\section{The two-flash threshold: An evaluation of critical-duration and visual-persistence hypotheses*}

\author{
DEAN G. PURCELL, $\dagger$ University of Toronto, Toronto, Ontario, Canada \\ and
}

ALAN L. STEWART, York University, Downsview, Toronto, Ontario, Canada

The two-flash threshold is reduced by increasing the duration of both pulses of light or light adapting the eye. Increasing the duration of the first pulse also decreases the two-flash threshold, contrary to what a critical-duration explanation of the threshold would predict. Decreasing the duration of the second pulse increases the threshold when the second pulse is very brief. Light adapting the eye under such conditions serves to increase the two-flash threshold, unlike the effect adaptation has when a long second pulse is used.

Temporal resolution in the visual system has been measured using the two-flash threshold (Kietzman, 1967; Lewis, 1967, 1968). This technique involves the successive presentation of two pulses of light to the same retinal locus. Two pulses of light presented temporally, such that they lie below the two-flash threshold, are not resolved and are seen as one flash. The measure of the two-flash threshold is taken as the temporal interval from offset of the first pulse to onset of the second pulse. This interval is referred to as the interflash interval.

One explanation of the two-flash threshold utilizes the concept of critical duration for temporal integration of visual stimuli (Davey, 1952). According to the critical-duration hypothesis, pulses of light that cannot be resolved must fall within the critical duration and are processed as one input. Lewis (1967) infers that the two-flash threshold may yield the critical duration directly.

Another interpretation of the failure of temporal resolution at the two-flash threshold has been proposed. Wundt (as reported by Sperling, 1960) argued that the failure to resolve two puises of light was the result of the second pulse exciting the visual system before the neural response to the first stimulus had ceased. This hypothesis does not provide that the total energy from two pulses is integrated in the visual system. More recently, Pollack, Ptashne, and Carter (1969) used the persistence hypothesis to account for age differences in the two-flash threshold.

The idea that the response to a brief visual stimulus lasts longer than does the

* This rescarch was supported by the National Institutes of Health IGrant NB-07622-02 to W. N. Dembers. The preparation of this report Wa upported by the National Rescarch Council of (anada (Grant APA 191 to Marilyn (. Simith) and by the Defense Research Board of Canada (Cirant 9401-38 to Marilyn C. Smith).

Now at the New School for Social Research. 66 West 12 ih Street. New York, New York 10011. stimulus itself has been well documented. Wicke, Donchin, and Lindsley (1963) confirmed this for the evoked potential; and Mackworth (1963), Sperling (1960), and Haber (1968) for conscious visual experience. In this paper, visual persistence will refer to the phenomenal manifestation of a visual stimulus that outlasts the physical duration of that stimulus. Visual persistence has the same figure-ground relationship as the stimulus that generated it and appears to dim as a function of time from offset of stimulation (Haber \& Standing, 1969).

Recently the duration of visual persistence has been related to the duration and luminance of the stimulus that generated it. Haber and Standing (1969) report that an outline of a black circle presented repeatedly, on for 4 to $200 \mathrm{msec}$ and then off for some period, persists for about $250 \mathrm{msec}$. They report that persistence was longer for low- as compared to high-luminance exposures. Persistence could also be increased by reducing the intensity of the adapting field. Increases in stimulus duration did not change estimates of persistence. However, Standing, Haber, Cataldo, and Sales (1969) found that increasing stimulus duration above recognition threshold values did decrease persistence. In another experiment by Haber and Standing (as reported by Haber, 1968), Os were trained to adjust a click to coincide with the onset of a flash and another to coincide with its offset. When the flash was brief ( 5 to $50 \mathrm{msec}$ ), the interclick interval was about $150 \mathrm{msec}$ longer than the flash. However, when the flash was long ( 200 to $300 \mathrm{msec}$ ), the interclick interval was always the same as the flash duration. just as though no visual persistence was associated with the longer flash. Thus. increasing the luminance or duration of a stimulus, as well as increasing the luminance of its adapting field, may reduce its visual persistence.
Those manipulations which serve to decrease estimates of visual persistence have also been shown to reduce the two-flash threshold. Dunlap (1915), Mahneke (1958), and Kietzman (1967) demonstrated that increasing the duration of the individual light pulses reduces the two-flash threshold. Lewis (1967) also reported that increasing the intensity of the individual pulses reduces the two-flash threshold. Dunlap (1915) found that presenting his stimuli to the light-adapted eye yielded shorter two-flash thresholds than presenting the same stimuli to the dark-adapted eye.

An analysis of the two-flash threshold based on visual persistence would provide that when two pulses of light are presented below the two-flash threshold the visual persistence of the first pulse provides a phenomenal link with the second pulse. That is, if the second pulse is presented before the visual persistence associated with the first pulse has dimmed sufficiently, the $O$ will see only one flash. On the other hand, if the second pulse excites the visual system after the persistence has dimmed, the $\mathrm{O}$ will see two flashes. We would like to point out that Haber's work on visual persistence has been concerned with its absolute duration. We do not mean to suggest that the two-flash threshold can be used to measure this absolute duration. All that should be necessary for an $\mathrm{O}$ to report seeing two flashes, under the persistence hypothesis, is that the persistence dim sufficiently so that a change in brightness over time is detectable. Specifically, we predict that operations which serve to decrease the brightness of visual persistence from the first pulse will, in so doing, lower the two-flash threshold. In this paper two such operations are used: increasing the duration of the first pulse and presenting the pulses on a bright adaptation field.

Four experiments were run: Experiment 1 was designed to verify previous experimental findings on the two-flash threshold, and Experiments 2-4 were designed to test the critical-duration and visual-persistence hypotheses.

\section{APPARATUS AND STIMULI}

The stimuli consisted of a white backlighted circular disk, with a luminance of $40 \mathrm{fL}$, subtending $38 \mathrm{~min}$ of visual arc. A single pulse of this disk was clearly above detection threshold in all four experiments. The disk was presented foveally in all experiments. The fixation field was defined by four peripheral points of dim red light arranged in a diamond pattern. All viewing was monoptic with the right eye. Each stimulus presentation consisted of two pulses of the disk. The duration of each 


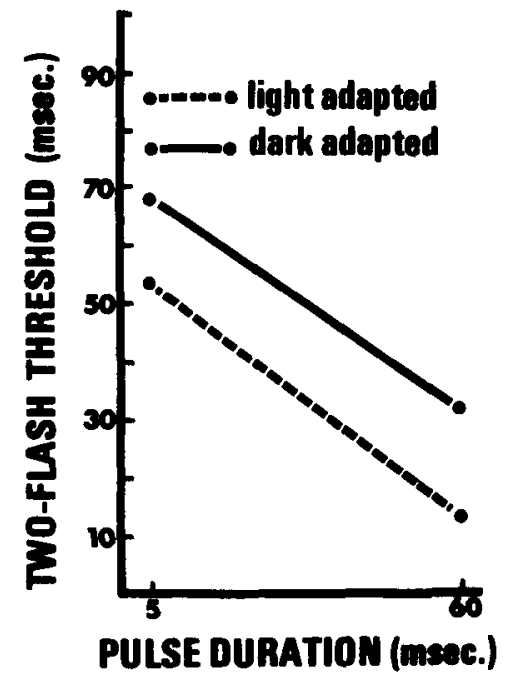

Fig. 1. The two-flash threshold as a function of pulse duration and state of light adaptation, averaged over six $O$ s.

individual pulse of light and the interval separating them was controlled by a Scientific Prototype tachistoscope, Model GB. This unit also provided a continuous white light-adapting field ( 180 min square) upon which pulses were superimposed in conditions calling for light adaptation. When this field was on, it had a luminance of $25 \mathrm{fL}$.

\section{METHOD}

A method-of-limits technique consisting of alternating ascending and descending series of interpulse intervals, in 5-msec steps, was used to determine two-flash thresholds. The threshold for each $O$ was taken as the mean point of transition of response: yes to no, no to yes, averaged over each series in a given condition. Os were instructed to respond "Yes" whenever the presentation of the two pulses appeared to flicker and "No" when they appeared steady throughout their exposure. A yes response was taken to indicate that the temporal interval separating the two pulses was above the two-flash threshold. A no response was taken to indicate that the interval was below the two-flash threshold.

In all experiments Os were light or dark adapted, as the experimental condition required, for $10 \mathrm{~min}$ before that condition was run. At the beginning of each experiment, naive Os were given practice to familiarize them with the task. No feedback was given during practice or during the experiment.

\section{EXPERIMENT 1}

This experiment was designed to investigate the effect on the two-flash threshold of increasing the duration of the individual pulses of light, as well as the effect of light adapting the visual system. Previous findings demonstrate that both of these procedures decrease the two-flash threshold (Dunlap, 1915; Mahneke, 1958; Kietzman, 1967). Six Os were run under each of four conditions in a 2 by 2 repeated-measures design. The order of presentation of each condition was randomized for each $\mathrm{O}$. Two conditions of individual pulse duration consisting of two pulses, each $5 \mathrm{msec}$ long, and two pulses, each $60 \mathrm{msec}$ long, were presented under both light and dark adaptation. Six ascending and descending series were run on each $\mathrm{O}$, under each of the four conditions, to determine the two-flash threshold.

\section{Results and Discussion}

The two-flash threshold is reduced by increasing the duration of the individual pulses $[F(1,5)=334.96, p<.01]$ regardless of the state of adaptation. Light adapting the visual system also reduces the two-flash threshold $[F(1,5)=18.39$, $p<.01$; see Fig. 1].

These findings are consistent with previous findings that the two-flash threshold is reduced by light adapting the visual system or increasing the duration of the individual pulses. The fact that both operations which have been shown to decrease visual persistence serve to decrease the two-flash threshold supports a visual persistence interpretation of the two-flash threshold.

\section{EXPERIMENT 2}

A second experiment was specifically designed to test the critical-duration hypothesis of the two-flash threshold against the visual-persistence hypothesis. If the critical-duration hypothesis applies, the important variable in manipulation of the two-flash threshold is the total duration of the two pulses. Changing the duration of one pulse relative to the other should not change the two-flash threshold as long as the total duration of the pulses is unaltered. On the other hand, experiments on visual persistence suggest that increasing the duration of the first pulse relative to the second should reduce the persistence of the first and lower the two-flash threshold. Thus, a visual persistence hypothesis predicts that when the total duration of the two pulses is held constant and their relative durations are changed, the two-flash threshold will be lower for those stimulus presentations where the longer pulse occurs first. Five Os were run under each of six experimental conditions in a 2 by 3 repeated-measures design. Under those conditions where the first and second pulse were not of equal duration, the total

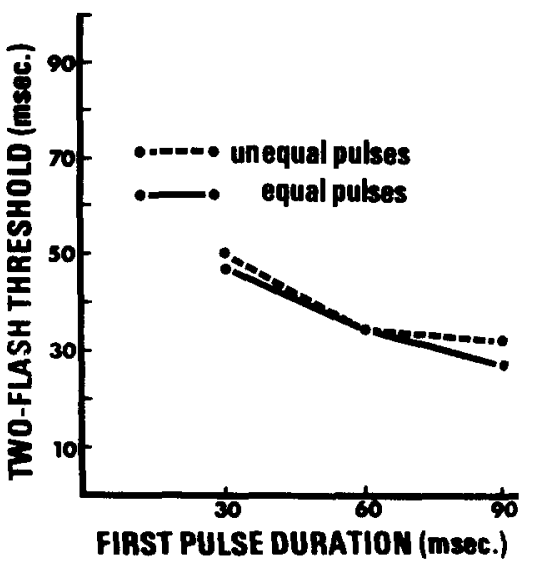

Fig. 2. The two-flash threshoid as : function of first pulse duration comparin equal pulse pairs with unequal pulse pairs averaged over five 0 s.

duration of the two pulses was helc constant at $120 \mathrm{msec}$. The pulse pair: consisted of individual pulses 30 anc $90 \mathrm{msec}, 60$ and $60 \mathrm{msec}$, and 90 anc $30 \mathrm{msec}$. Three other conditions were rur where the length of both pulses was sel equal to the duration of the first pulse ir one of the other three conditions. That is 90 and $90 \mathrm{msec}, 60$ and $60 \mathrm{msec}$, and 30 and $30 \mathrm{msec}$. The last three conditions were run in an effort to discover any possible effect the duration of the second pulse might have on the two-flash threshold. Twelve ascending and descending series were run on each $\mathrm{O}$ unde1 each of the six conditions.

\section{Results and Discussion}

The effect of the duration of the firs 1 pulse is significant $[F(2,8)=112.37$. $\mathrm{p}<.01]$, with the two-flash threshold decreasing as the duration of the first pulse is increased. This finding supports a visual persistence hypothesis. However, it migh1 be argued that these results do support a critical-duration hypothesis if it is assumed that integration within the critical duration begins at the onset of the first pulse. Under this assumption, as the first pulse duration is increased, the second pulse must be presented at a shorter interflash interval in order for its onset to fall within the critical duration. This implies that as the first pulse duration is increased a like decrease in the interflash interval will keep the first pulse and the onset of the second pulse within the critical duration and hence below the two-flash threshold. Figure 2 demonstrates that this is not the case. The absolute decrease in the two-flash threshold is not predicted by the absolute increase in first pulse duration.

Mahneke (1958) proposed that the total quantity of light in the two pulses determined the two-flash threshold. In one 


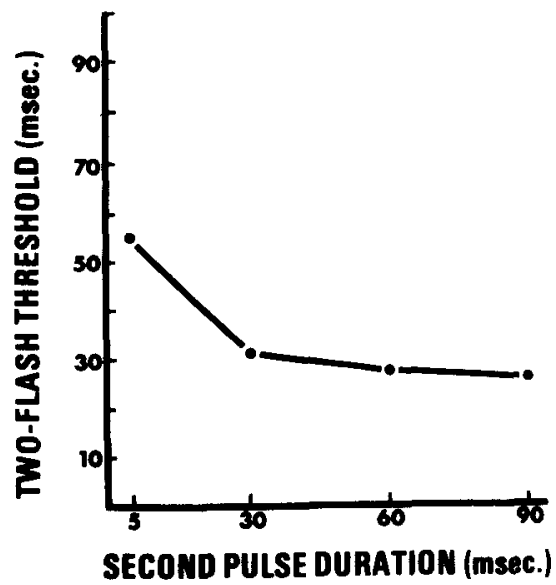

Fig. 3. The two-flash threshold as a function of second pulse duration with first pulse duration held constant at $90 \mathrm{msec}$, averaged over five Os.

condition of Experiment 2, the total quantity of light in the two pulses was held constant at $40 \mathrm{fL}$ exposed for $120 \mathrm{msec}$. In spite of this, the two-flash threshold was found to decrease as the first pulse duration increased. This is not consistent with Mahneke's proposal that the two-flash threshold is reduced only as a consequence of increasing the total quantity of light in the two pulses.

The effect of the duration of the second pulse is also significant $[F(1,4)=14.34$, $\mathrm{p}<.025$; see Fig. 2]. This effect appears to be due primarily to the $90-90-\mathrm{msec}$ vs the 90-30-msec pulse pair comparison. It appears that reducing the duration of the second pulse can increase the two-flash threshold when the duration of the first pulse is relatively long. Substantiation of this finding would indicate a limitation of a simple visual persistence hypothesis.

\section{EXPERIMENT 3}

A third experiment was designed to investigate the possibility that decreasing the duration of the second pulse can increase the two-flash threshold. Five Os were run under each of four conditions in a one-way repeated-measures design. The duration of the first pulse was held constant at $90 \mathrm{msec}$, and the second pulse was $90,60,30$, or $5 \mathrm{msec}$ long, as the condition required. The stimuli were presented to the dark-adapted eye for eight ascending and descending series under each condition.

\section{Results and Discussion}

The interflash interval corresponding to the two-flash threshold is increased as the duration of the second pulse is decreased $[F(3,12)=54.27, p<.01$; see Fig. 3]. This finding is not consistent with a visual-persistence hypothesis. Changes in the duration of the second pulse should not influence persistence of the first pulse and thus should not change the two-flash threshold.

\section{EXPERIMENT 4}

Increasing the duration of the first pulse decreases the two-flash threshold when long second pulses are used. However, when the duration of the second pulse is reduced, the threshold value increases. We have also found that with relatively long pulses light adaptation reduces the two-flash threshold. It would be of interest to determine the effect of light adaptation on a two-pulse presentation consisting of a long first pulse and a short second pulse. Light adaptation should reduce visual persistence and lower the two-flash threshold. To test this, six Os were run under each of four conditions in a 2 by 2 repeated-measures design. The duration of the first pulse was held constant at $90 \mathrm{msec}$. The duration of the second pulse was $90 \mathrm{msec}$ in one condition and $2 \mathrm{msec}$ in the other. The Os were run under each of these conditions in both the light-adapted and dark-adapted states. Eight ascending and descending series were run on each $\mathrm{O}$ under each of the four conditions.

\section{Results and Discussion}

Reducing the duration of the second pulse from $90 \mathrm{msec}$ to $2 \mathrm{msec}$ significantly increased the two-flash threshold $[F(1,5)=83.28, p<.01]$. This finding is consistent with Experiment 3 . The overall effect of light adaptation was not significant, but the interaction of second pulse duration with adaptation state was $[F(1,5)=24.64, p<.01$; see Fig. 4] . Light adapting long equal-duration pulses decreased the two-flash threshold, while light adapting a long first pulse and a short second pulse increased the two-flash threshold. The results of Experiment 4 question both the visual persistence and the critical-duration hypothesis of the two-flash threshold. Light adaptation reduces visual persistence (Haber \& Standing, 1969; Standing, Haber, Cataldo, \& Sales, 1969) and should lower the two-flash threshold regardless of the duration of the second pulse. Light adaptation reduces the critical duration, as measured by brightness estimates (Keller, 1941), and should result in a reduction of the two-flash threshold no matter what the duration of the second pulse.

\section{GENERAL DISCUSSION}

The four experiments presented in this paper provide that increasing the duration of the first pulse as well as light adaptation serve to reduce the two-flash threshold.

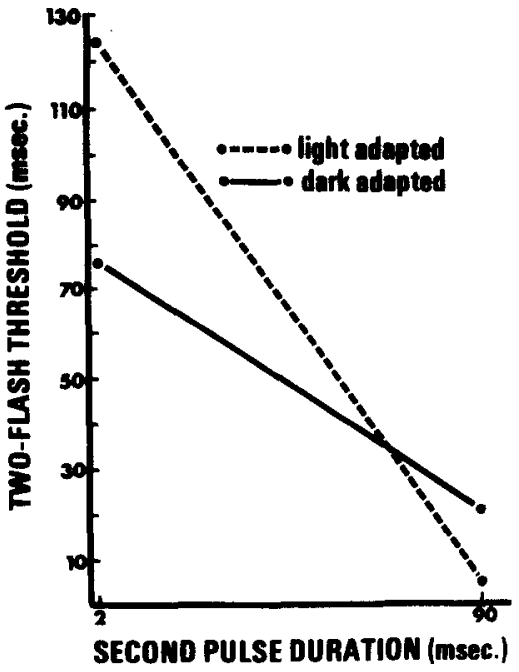

Fig. 4. The two-flash threshold as a function of second pulse duration and state of light adaptation with first pulse duration held constant at $90 \mathrm{msec}$, averaged over six Os.

However, decreasing the duration of the second pulse increases the two-flash threshold under these conditions. We have pointed out that reference to a critical-duration hypothesis will not allow interpretation of Experiments 2 and 4. Similarly, Experiments 3 and 4 cannot be explained strictly in terms of visual persistence.

We would like to suggest an analysis of these experiments based on the concept of lateral inhibition as derived from work on Limulus (Hartline, 1969; Ratliff, 1965). Recent and various applications of the concept of lateral inhibition to human visual perception suggest such an analysis. Figural aftereffects (Ganz, 1966), backward masking (Purcell, Stewart, \& Dember, 1968; Weisstein, 1968), rapid light and dark adaptation (Dowling, 1967), and flicker fusion (Ratliff, Knight, Toyoda, \& Hartline, 1967; Fiorentini \& Ma ffei 1968) have all been analyzed largely in terms of lateral inhibitory interactions. Since in the present studies we did not vary the size of our stimulus disk, it might be argued that we were not manipulating lateral inhibition upon those elements stimulated by the disk. However, our disk was of sufficient size (diameter of $38 \mathrm{~min}$ ) so that a large number of adjacent visual receptors were stimulated during each target presentation. These stimulated elements could be expected to exert lateral inhibitory influences upon each other. To quote Hartline (1969): "The influences are mutual: each receptor, being a neighbor of its neighbors, inhibits and is inhibited by those neighbors."

Comparison of conditions that influence 
the two-flash threshold with those conditions that govern the amount of lateral inhibition present in a stimulated area of the visual system show the following similarities. Using psychophysical tasks, Dunlap (1915), Mahneke (1958), and Keitzman (1967) have demonstrated that increasing the duration of the individual light pulses reduces the two-flash threshold. Likewise, Lewis (1967) reports that increasing the intensity of the individual pulses also reduces the two-flash threshold. In the same way, within the visual system of the limulus both increasing the duration or the intensity of a visual stimulus serves to increase the amount of lateral inhibition generated by it (Ratliff, 1965).

Dunlap (1915) found that light adaptation reduced the two-flash threshold. Lewis (1968) found that increasing the size of the pulsed stimuli generally decreased the two-flash threshold. Both procedures have the effect of subjecting more and more elements of the visual system to stimulation. Increasing the size of the target increases the number of stimulated neural elements as the radius of the target increases, while light adaptation of the entire eye stimulates all elements equally. As there is spatial summation of lateral inhibition (Hartline, 1969), the overall level of inhibition in the area stimulated by the disk should be increased, with in limits, by increasing the disk size or the level of light adaptation.

Inhibition in a stimulated area of the visual system will decrease the effectiveness of a given stimulus presented to that area. In terms of our argument, the inhibition produced by a given stimulus, such as the first pulse of a two-pulse sequence, reduces the effectiveness of that stimulus in producing visual persistence. That is, the inhibition generated by a given stimulus serves to damp out the activity that, if no inhibition were present, would continue after the stimulus offset. The greater the amount of inhibition -due to an increase in target size, duration, luminance, or the statc of light adaptation-the less will be the effectiveness of a given stimulus in producing visual persistence to bridge the interpulse interval. Thus, the two-flash threshold should decrease as inhibition is increased. Experiments 3 and 4 indicate an important qualification to this argument. The second pulse must either be of sufficient magnitude to overcome inhibition that outlasts stimulation by the first pulse, or a sufficient interval must exist between the pulses to allow inhibition to decay. If the pulse is not of sufficient magnitude, then it, like visual persistence, will be damped out.

Lateral inhibition has been widely implicated as the mechanism underlying the sharpening and definition of visual spatial contours (Ratliff, 1965). Ratliff, Knight, Toyoda, and Hartline (1967) argue that lateral inhibition also provides a mechanism for the enhancement of significant variations in temporal patterns of illumination. The present research is consistent with the second argument and extends it to human Os and the two-flash threshold.

\section{REFERENCES}

DAVEY, E. The intensity-time relationship for multiple flashes of light in the peripheral retina. Journal of the Optical Society of America, 1952, 42, 937-941.

DOWL.ING, 3. E. The site of visual adaptation. Science, $1967,155,273-279$.

DUNLAP, K. The shortest perceptible time-interval between two tlashes of light. Psychological Review, 1915, 22, 226-250.

IIORFNTINI, A., \& MAFrEI, L. Perceptual correlates of inhibitory and facilitatory spatial interactions in the visual system. Vision Rescarch. 1968.8.1195-1203.

GANZ, L. Mechanism of the figural aftereffects. Psychological Review, 1966, 73, 128-150.

HABER, R. N. Temporal integration of suprathreshold perceptual processes. Paper read at the meetings of the American Psychological Association, August 1968.

HABIE, R. N., \& STANDING, L. G. Direct measures of short-term visual storage. Quarterly Journal of Experimental Psychology. 1969, 21.43-54.
HARTLINE, H. K. Visúal receptors and retin interaction. Science, 1969, 164, 270-278.

KELLLER, M. The relation between the critic duration and intensity in brightne discrimination. Journal of Experiment Psychology, 1941, 28, 407-418.

KIETZMAN, M. L. Two-pulse measures , temporal resolution as a function of stimul encrgy. Journal of the Optical Socicty America, 1967, 57, 809-813.

LEWIS, M. F. Two-flash thresholds as a functic of luminance in the dark adapted eyc. Journ of the Optical Society of Amcrica, 1967, 5 814-815.

IEWIS, M. l:. Two-flash thresholds as a functio of flash luminance and area. Perception. Psychophysics, 1968, 4, 241-244.

MACKWORTH, J. E. The duration of the visu image. Canadian Journal of Psychology, 196 $17,62-81$.

MAHNEKE, A. Foveal discrimination measure with two successive light fashes: psychophysical study. Acta Ophthamologic 1958, 36, 3-11.

POLLACK, R. H., PTASHNE, R. I., \& CARTE, D. J. The effects of age and intelligence on th dark-interval threshold. Perception Psychophysics, 1969, 6, 50-52.

PURCELL, D. G., STEWART, A. L, DEMBER, W. N. Spatial effectiveness of th mask: Lateral inhibition in visual backwat masking. Perception \& Psychophysics, 1968. 344-346.

RATLIFF, F. Mach bands: Quantitative studit on neural networks in the retin San Francisco: Holden Day, 1965.

RATLIFI, I., KNIGHT, B. W., TOYODA, J., HARTLINE, H. K. Enhancement of tlicker b lateral inhibition. Science, 1967, 15: 392-393.

SPI:RLING. G. The information available in bric visual presentations. Psycholnate: Monographs. 1960, 74, 1-29.

STANDING, L. G.. HABIER, R. N., (ATALDC M., \& SALISS, D. B. Two types of short-ter visual storage. Perception \& Psychophysic $1969,5,193-196$.

WIIISSTEIN, N. A Rashersky-Landaht neur: net: Simulation of metacontrast. Psychologici Review, 1968, 75, 494-521.

WICKE, J. D., DONCHIN, E.. \& LINDSLIEY, [ B. Visual evoked potential as a function $c$ flash luminance and duration. Science. 1966 146, 83-85.

(Accepted for publication April 24. 1970.) 